\title{
新型吡咯类氨基酸缀合物的合成与抗肿瘤活性测定
}

\author{
李衍忠赵萍詹晓平刘增路毛振民* \\ (上海交通大学药学院 上海 200240)
}

\begin{abstract}
摘要 以苯甲醛或 3,4-二甲氧基苯甲醛为原料经 Wittig-Horner 反应、Van Leusen 吡咯合成法、水解等多步反应，设计 并合成了 16 个未见文献报道的吡咯类氨基酸缀合物 7a $\sim 7 \mathbf{p}$. 其结构经 ${ }^{1} \mathrm{H} N M R,{ }^{13} \mathrm{CNMR}$ 及 HRMS 确证. 采用噻唑蓝 (MTT)法测试了目标化合物对 MCF-7, MGC80-3, Hep G2, CT-26 及 HUVEC 细胞的增殖抑制作用. 结果表明, 几乎所有 化合物对人体正常细胞 HUVEC 无明显抑制作用，化合物 7a $\sim 7 \mathbf{h}$ 对 Hep G2 细胞株有一定的抑制活性，化合物 7i $\sim 7 \mathbf{p}$ 对 MCF-7 及 MGC80-3 较强的抑制活性. 其中化合物 7o 7p 对 MCF-7 细胞株的抑制活性最强.
\end{abstract}

关键词 吡咯; 氨基酸; 合成; 抗肿瘤活性

\section{Synthesis and Cytotoxic Activities of Novel Amino Acid-Conjugates of Pyrrole Derivatives}

\author{
Li, Yanzhong Zhao, Ping Zhan, Xiaoping Liu, Zenglu Mao, Zhenmin* \\ (School of Pharmacy, Shanghai Jiaotong University, Shanghai 200240)
}

\begin{abstract}
Sixteen novel amino acid-conjugates of pyrrole derivatives $\mathbf{7} \mathbf{a} \sim \mathbf{7} \mathbf{p}$ were designed and synthesized using benzaldehyde or 3,4-dimethoxybenzaldehyde as raw materials. The compounds were synthesized via multi-step reaction including Wittig-Horner, Van Leusen and hydrolysis. The structures of all the target compounds were characterized by ${ }^{1} \mathrm{H}$ NMR, ${ }^{13} \mathrm{C}$ NMR and HRMS. Moreover, the cell proliferation inhibiting activities of the target compounds were evaluated against MCF-7, MGC80-3, Hep G2, CT-26 and HUVEC cell lines by thiazolyl blue tetrazolium bromide (MTT) method. The result indicated that most of the compounds had no significant inhibitory effect against normal human cell HUVEC. However compounds $\mathbf{7 a} \sim \mathbf{7 h}$ showed significant inhibitory effect against Hep G2, compounds $\mathbf{7 i} \sim \mathbf{7 p}$ showed stronger inhibitory effect against MCF-7 and MGC 80-3, and compound 7o $\sim \mathbf{7 p}$ exhibited the strongest inhibitory activity against MCF-7 in all of the compounds.
\end{abstract}

Keywords pyrrole; amino acid; synthesis; cytotoxic activities

吡咯类化合物是杂环化学研究领域中的重要组成 部分 ${ }^{[1]}$. 自 1857 年 Anderson 从骨胶油中分离出吡咯以 来, 吡咯化学就随着大量取代吡咯类化合物从天然产物 中的分离而不断发展 ${ }^{[2 \sim 4]}$. 吡咯的衍生物极为丰富, 很 多有重要生理作用的物质都是由吡咯衍生物组成的, 如 叶绿素、血红蛋白、维生素 B12 等都是吡咯的衍生物, 所 以对吡咯衍生物生理作用上价值的探索一直备受人们 关注. 在过去的几十年中, 科研工作者们对吡咯衍生物 的合成及其药效作用也进行了广泛的研究 ${ }^{[5 \sim 9]}$. 其中 3,4-二取代吡咯的合成以及其作为抗炎、抗病毒、抗真 菌药物的研究已见诸多报道 ${ }^{[10 ~ 12]}$. 我们课题组报道了
3,4-二取代吡咯衍生物的抗肿瘤活性 ${ }^{[13,14]}$, 当 4 位是 3氯-4-氟苯基取代，或 4-甲颈基苯基取代时，得到的系列 化合物具有显著的抗肿瘤活性, 部分化合物甚至表现出 与紫杉醇相近的活性, 而且令人欣喜的是这些化合物对 正常细胞几乎没有抑制活性，这显示了此类化合物具有 较高的选择性及安全性. 通过对文献中的化合物以及我 们课题组的前期研究工作进行构效关系总结, 发现: 当 保持吡咯环 1 位无取代基, 3 位为羰基、硝基等吸电子基 团，吡咯环 4 位为苯环尤其是苯环上含有甲基、甲氧基、 甲統基等供电子基团时，化合物往往具有较好的抗肿瘤 活性. 本研究将继续开展对 3,4-二取代吡咯的研究, 探

\footnotetext{
*E-mail: zmmao@sjtu.edu.cn

Received July 10, 2014; revised August 20, 2014; published online September 12, 2014.

Project supported by the National Science and Technology Major Special Drug Discovery (No. 2010ZX09401404-004).

国家 “重大新药创制” 科技重大专项(No. 2010ZX09401404-004)资助项目.
} 
索新类型的化合物的抗肿瘤活性. 据研究报道 ${ }^{[15]}$ 肿瘤 细胞对氨基酸的需求不同于正常细胞. 肿瘤组织为了蛋 白质合成和细胞增生, 需要不断摄取多种氨基酸为肿瘤 细胞增殖提供必需的营养物质, 其对氨基酸的需求要远 高于正常细胞. 将氨基酸引入抗肿瘤药物分子中, 可以 提高其对肿瘤细胞的选择性、增强药物的脂溶性、缓解 药物对正常细胞的毒害作用 ${ }^{[16,17]}$, 这也为寻找和发现高 效、低毒抗肿瘤药物提供了新的重要方向 ${ }^{[18-20]}$. 我们尝 试当 4 位为苯基或 3,4-二甲氧基苯基取代时，以 3 位羰 基作为桥接, 在 3 位引入不同的氨基酸, 以期得到有价 值的化合物. 本研究共合成了 16 个未见报道的化合物, 其结构经 ${ }^{1} \mathrm{H} \mathrm{NMR},{ }^{13} \mathrm{C} \mathrm{NMR}$ 及 HRMS 表征, 并选择 5 种细胞株, 分别为 MCF-7(人乳腺癌细胞), MGC80-3(人 胃癌细胞), Hep G2(人肝癌细胞), CT-26(小鼠结肠癌细 胞)和 HUVEC(人脐静脉内皮细胞)对目标化合物进行了 抗肿瘤活性的篮选. 合成路线见 Scheme 1.

\section{1 结果与讨论}

\section{1 化合物的合成}

以磷酸三乙酯和芳香醛为原料, 先通过 Wittig-Horner 反应生成 $\alpha, \beta$-不饱和烯烃化合物 $\mathbf{3 a} \sim \mathbf{3 b}$, 再利用 Van Leusen 合成法实现吡咯环的构建, 在碱性下 水解、酸化吡咯环上的酯基，接着以 1-乙基-(3-二甲基
氨基丙基)碳酰二亚胺盐酸盐(EDCI)和 1-羟基苯并三唑 (HOBT) 作为缩合剂，与取代的氨基酸甲酯进行缩合反 应得到化合物 $6 \mathrm{a} \sim 6 \mathrm{p}$, 在碱性下水解、酸化, 脱甲氧基 后得到目标化合物 $\mathbf{7 a} \sim \mathbf{7 p}$. 反应中使用的 Van Leusen 法是一种制备 3,4-二取代吡咯类化合物简便、高效的方 法，其中以使用对甲苯磺酰基甲基异腈(Tosmic)的 Van Leusen 合成法最为普遍, 此法首先要完成 $\alpha, \beta$-不饱和烯 烃的制备. 根据目标化合物的结构特点, 想要接上氨基 酸, 要先在吡咯环的 3 号位引入酯基, 而酯基的吸电性 较弱, 直接和芳香醛反应制备不饱和烯烃比较困难, 所 以对于酯基相应的 $\alpha, \beta$-不饱和烯烃的合成, 我们选择使 用活性更高的磷脂叶丽德试剂进行 Wittig-Horner 反应 来制备. 在得到化合物 $5 \mathbf{a} \sim \mathbf{5 b}$ 后，最初设想是将羧基转 化为活性更高的酰氯和氨基酸反应，但实验过程中发 现，制备酰氯的反应条件比较严苛，反应后呈胶状物， 纯化比较困难，会对其后的反应造成困难. 之后我们选 用 EDCI 和 HOBT 的缩合剂组合，这种方法要先将氨基 酸的羧基变成酯基进行保护, HOBT 的引入可以在活化 吡咯羧基的同时有效避免氨基酸的消旋. 该缩合常温下 即可进行且反应迅速，后处理简单，收率高 $(65 \%$ 95\%).

\section{2 生物活性}

化合物 7a $\sim \mathbf{p}$ 对 5 种细胞株的增殖抑制作用如表 1<smiles>[R]c1ccc(/C=C/C(=O)OCC)cc1[R]</smiles><smiles>[R]c1ccc(-c2c[nH]cc2C(=O)OCC)cc1[R]</smiles>

4
(1) $\mathrm{EtOH}, \mathrm{NaOH}$, reflux

(2) $\mathrm{HCl}$<smiles>[R]c1ccc(-c2c[nH]cc2C(=O)O)cc1[R]</smiles>

5

2a $\sim 5 a: \mathrm{R}^{1}=\mathrm{H}, \mathrm{R}^{2}=\mathrm{H}$;

$2 b \sim 5 b: R^{1}=O M e, R^{2}=O M e$<smiles>[R]c1ccc(-c2c[nH]cc2C(=O)NC([R])C(=O)OC)cc1[R]</smiles>

(1) $\mathrm{NaOH}$, r.t.

(2) $\mathrm{HCl}$<smiles>[R]c1ccc(-c2c[nH]cc2C(=O)NC([R3])C(=O)O)cc1[R]</smiles>

6a $\sim 6 h, 7 a \sim 7 h: \mathrm{R}^{1}=\mathrm{H}, \mathrm{R}^{2}=\mathrm{H} ; \mathrm{R}^{3}=$ (a) $\mathrm{H},(\mathbf{b}) \mathrm{CH}_{3}$ (c) $\mathrm{CH}\left(\mathrm{CH}_{3}\right)_{2}$, (d) $\mathrm{CH}_{2} \mathrm{CH}\left(\mathrm{CH}_{3}\right)_{2},(\mathbf{e}) \mathrm{CH}_{2} \mathrm{Ph}$,

(f) tetrahydropyrrol-2-yl, (g) $\mathrm{CH}_{2} \mathrm{OH}$, (h) $\mathrm{CH}_{2} \mathrm{COOH}$

6i $\sim 6 p, 7 \mathbf{i} \sim 7 \mathbf{p}: \mathrm{R}^{1}=\mathrm{OMe}, \mathrm{R}^{2}=\mathrm{OMe} ; \mathrm{R}^{3}=$ (i) $\mathrm{H},(\mathbf{j}) \mathrm{CH}_{3}(\mathbf{k}) \mathrm{CH}\left(\mathrm{CH}_{3}\right)_{2}$, (I) $\mathrm{CH}_{2} \mathrm{CH}\left(\mathrm{CH}_{3}\right)_{2}$,

(m) $\mathrm{CH}_{2} \mathrm{Ph}$, (n) tetrahydropyrrol-2-yl, (o) $\mathrm{CH}_{2} \mathrm{OH},(\mathbf{p}) \mathrm{CH}_{2} \mathrm{COOH}$

\section{Scheme 1}


表 1 目标化合物对细胞株增殖抑制活性结果 $\left[\mathrm{IC}_{50} /\left(\mu \mathrm{mol} \cdot \mathrm{L}^{-1}\right)\right]^{a}$

Table 1 Results of anti-proliferative activity of the target compounds on cells $\left[\mathrm{IC}_{50} /\left(\mu \mathrm{mol} \cdot \mathrm{L}^{-1}\right)\right]$

\begin{tabular}{|c|c|c|c|c|c|}
\hline Compd. & MCF-7 & MGC80-3 & Hep G2 & CT-26 & VEC \\
\hline $7 a$ & $>1000$ & $>1000$ & $>1000$ & $>1000$ & $>1000$ \\
\hline $7 b$ & 360.09 & $>1000$ & 217.91 & $>1000$ & $>1000$ \\
\hline $7 c$ & 322.48 & $>1000$ & 61.96 & $>1000$ & $>1000$ \\
\hline $7 d$ & 114.76 & $>1000$ & 247.61 & $>1000$ & $>1000$ \\
\hline $7 e$ & $>1000$ & $>1000$ & $>1000$ & $>1000$ & $>1000$ \\
\hline $7 f$ & $>1000$ & $>1000$ & $>1000$ & $>1000$ & $>1000$ \\
\hline $7 \mathrm{~g}$ & $>1000$ & $>1000$ & 130.63 & $>1000$ & $>1000$ \\
\hline $7 \mathrm{~h}$ & $>1000$ & $>1000$ & 466.45 & $>1000$ & $>1000$ \\
\hline $7 i$ & $>1000$ & 173.84 & 541.81 & $>1000$ & $>1000$ \\
\hline $7 \mathbf{j}$ & 306.34 & 200.55 & 173.31 & $>1000$ & $>1000$ \\
\hline $7 k$ & 266.68 & 360.03 & 115.12 & $>1000$ & $>1000$ \\
\hline 71 & 102.15 & 386.01 & 169.94 & $>1000$ & $>1000$ \\
\hline $7 \mathrm{~m}$ & $>1000$ & $>1000$ & $>1000$ & $>1000$ & 945 \\
\hline $7 n$ & $>1000$ & $>1000$ & $>1000$ & $>1000$ & $>1000$ \\
\hline 70 & 31.32 & 78.82 & 89.34 & $>1000$ & $>1000$ \\
\hline $7 p$ & 34.23 & 82.05 & 90.92 & $>1000$ & 732.55 \\
\hline 紫杉醇 & 3.06 & 4.2 & 5.7 & 9.7 & 68.9 \\
\hline
\end{tabular}

${ }^{a}$ Do not calculate $\mathrm{IC}_{50}$ value which is bigger than $1000 \mu \mathrm{mol} / \mathrm{L}$, described as $>1000$.

所示, 从表中结果可以看出: (1)绝大多数化合物对人体 正常细胞(HUVEC)没有明显的抑制作用, 所有化合物 对 CT-26 均无明显抑制活性, 这体现了该类化合物具有 较好的选择性和安全性. (2) 4 位为苯基取代和 4 位为 3,4-二甲氧基苯基取代的两个系列化合物相比较, 前者 的抗肿瘤活性较弱, 其只对 MCF-7 和 Hep G2 有一定的 抑制活性, 其中尤以 7d 对 MCF-7, 7c 对 Hep G2 有较强 的抑制能力, $\mathrm{IC}_{50}$ 值分别为 114.76 和 $61.96 \mu \mathrm{mol} \cdot \mathrm{L}^{-1}$; 后 者对 MCF-7, MGC80-3, Hep G2 的抑制作用相对来说较 好, 这说明苯环上供电子基团的引入可增强化合物的抗 肿瘤活性, 推究其原因可能与化合物作用靶点的电子云 分布有关. 该部分可能参与结合靶点的缺电子区域, 所 以此处电子云密度的提高将促进化合物与靶点的紧密 结合. (3)对比 4 位为 3,4-二甲氧基苯基取代的系列化合 物的活性数据发现, $7 \mathrm{o}$ 和 $7 \mathrm{p}$ 对三种肿瘤细胞均有较好 的抑制活性, 尤其是对 MCF-7 而言, 两者的 $\mathrm{IC}_{50}$ 值分别 为 31.32 和 $34.23 \mu \mathrm{mol} \cdot \mathrm{L}^{-1}$. 从引入氨基酸结构上来看 $7 \mathrm{o}$ 和 $7 \mathrm{p}$ 引入的氨基酸是丝氨酸和天冬氨酸, 这两种氨 基酸与该系列其它氨基酸相比, 都含有极性基团(分别 为羟基和羧基), 极性基团的存在会增强化合物的水溶 性, 从而提高化合物的生物利用度; 同时, 羟基和羧基 有利于化合物分子与受体之间形成氢键, 氢键的存在有 可能加强药物分子同靶点的结合, 从而表现出更好的抑 制活性. (5)当脯氨酸或者苯丙氨酸被用来改造 3,4-二取 代吡咯类化合物时, 所得的化合物 7e, $7 \mathrm{f}, 7 \mathrm{~m}, 7 \mathrm{n}$ 基本无 抑制活性, 这说明空间位阻较大时不利于化合物的抗肿 瘤活性.

\section{2 结论}

为了寻找新型的小分子抗肿瘤药物, 本研究尝试将 氨基酸引入到吡咯衍生物上, 进而得到一系列新型吡咯 类化合物. 在确立了简单、高效的合成路线后, 本研究 共设计合成了 16 个新型吡咯衍生物, 均未见文献报道. 细胞实验结果显示, 所有化合物对正常细胞没有杀伤能 力, 对 CT-26 几乎没有作用, 这说明了该类化合物具有 较好的选择抑制性; 部分化合物具有较强的抗肿瘤活 性, 尤其是接入丝氨酸及天冬氨酸的化合物 $7 \mathrm{o}$ 和 $7 \mathbf{p}$, 对几种肿瘤细胞均有不错的抑制活性. 并且, 通过构效 关系总结发现吡咯环上 3 位、 4 位取代基的电子云密度 和空间位阻对化合物抗肿瘤活性都有重要的影响. 这为 寻找和发现活性更强、副作用更小的抗肿瘤小分子药物 提供了新的思路, 具有进一步研究的意义.

\section{3 实验部分}

\section{1 仪器与试剂}

化学实验部分: 柱色谱采用 200 300 目硅胶粉(青 岛微纳化工有限公司生产); 薄层色谱(TLC)采用硅胶 $\mathrm{GF}_{254}$ 薄层层析硅胶板 (烟台江友硅胶开发有限公司); 熔 点采用 SGWX-4 显微熔点仪测定(上海精密科学仪器有 限公司), 温度计未经校正; ${ }^{1} \mathrm{H}$ NMR 和 ${ }^{13} \mathrm{C}$ NMR 采用 Bruker Avance III 400 型或 Varian NMR300 核磁共振仪 测定, 以 $\mathrm{TMS}$ 为内标, $\mathrm{CDCl}_{3}$ 或 $\mathrm{DMSO}-d_{6}$ 为溶剂; 高分 辨质谱(ESI-HRMS)采用 Waters 公司 UPLC-Q-TOF-MS 测定. 所用试剂均为市售的分析纯或化学纯试剂, 所有 手性 $\alpha$-氨基酸为 $L$ 型, 四氢呋喃(THF)、二氯甲烷、甲 
醇用前经干燥处理，氯化亚砜用前新蒸;

细胞增殖抑制实验部分：无菌净化工作台(上海新 苗医疗器械制备有限公司)、 $\mathrm{CO}_{2}$ 培养箱(Thermo Forma Series II)、酶联免疫检测仪(Thermo MK3)、倒置显微镜 (Olympus IX71)、MCF-7(人乳腺癌细胞)、MGC80-3(人 胃癌细胞)、Hep G2(人肝癌细胞)、CT-26(小鼠结肠癌细 胞)及 HUVEC(人脐静脉血管内皮细胞)均购买自中国科 学院细胞库, 所用细胞培养基等试剂均为市售生物用实 验标准.

\section{2 化合物的合成部分}

\subsection{1 $L$-氨基酸甲酯盐酸盐的合成通法}

$50 \mathrm{~mL}$ 甲醇中, 冰盐浴降温至 $-10 \sim-15{ }^{\circ} \mathrm{C}$, 逐滴 滴加 $2.2 \mathrm{~mL}$ 氯化亚砜, $10 \mathrm{~min}$ 内加完. 取 $25 \mathrm{mmol} L$-氨 基酸加入此混合液中, 滴加过程当中保持温度在一 $10{ }^{\circ} \mathrm{C}$, 滴加完后保持 $40{ }^{\circ} \mathrm{C}$ 继续反应 $3 \mathrm{~h}$. 升温至回流反 应 $3 \mathrm{~h}$ 后转室温反应, TLC 监测, 狮三酮显色. 反应结束 后, 减压蒸除溶剂, 残留物重新溶解于 $25 \mathrm{~mL}$ 甲醇重新 溶解, 再减压旋干, 此过程重复 3 次, 剩余固体重结晶 $[V$ (甲醇 $): V($ 乙酸乙酯 $)=3: 1]$, 得到各 $L$-氨基酸甲酯 盐酸盐 ${ }^{[21]}$. $L$-氨基酸甲酯以盐酸盐的形式存在有利于其 保存，该反应收率 $90 \% \sim 97 \%$.

\subsection{2 磷酸三乙酯的合成}

向反应瓶中依次加入 $17.2 \mathrm{~mL}(0.963 \mathrm{~g} / \mathrm{mL}, 0.1 \mathrm{~mol})$ 磷酸三乙酯和 $10.6 \mathrm{~mL}(1.15 \mathrm{~g} / \mathrm{mL}, 0.1 \mathrm{~mol})$ 氯乙酸乙酯, 回流摚拌反应约 $4 \mathrm{~h}$, 至不再有气体(氯乙烷)放出. 减压 蒸馏收集 $112{ }^{\circ} \mathrm{C} / 400 \mathrm{~Pa}$ 馏分, 得无色透明液体 ${ }^{[22]}, 20.3$ $\mathrm{g}$, 收率 90.5\%. ${ }^{1} \mathrm{H} \mathrm{NMR}\left(300 \mathrm{MHz}, \mathrm{CDCl}_{3}\right) \delta: 4.18 \sim 4.07$ (m, 6H, $\left.\mathrm{OCH}_{2} \mathrm{CH}_{3}\right), 2.91$ (d, $J=21.3 \mathrm{~Hz}, 2 \mathrm{H}, \mathrm{CH}_{2} \mathrm{CO}$ ), $1.32 \sim 1.20\left(\mathrm{~m}, 9 \mathrm{H}, \mathrm{OCH}_{2} \mathrm{CH}_{3}\right)$.

\subsection{3 $3 \mathrm{a}$ 和 $3 \mathrm{~b}$ 的合成}

以 3a 为例: $60 \mathrm{mmol} t-\mathrm{BuOK}$ 加入 $100 \mathrm{~mL}$ 无水 $\mathrm{THF}$ 中, 搅拌, 逐滴加入 $55 \mathrm{mmol}$ 磷酸三乙酯, 滴毕, 搅拌 $30 \mathrm{~min}$, 加入 $50 \mathrm{mmol}$ 苯甲醛, 室温反应 $4 \mathrm{~h}$, TLC 监测 反应完毕后, 减压旋蒸除去 THF, 加水 $100 \mathrm{~mL}$, 乙酸乙 酯萃取 $(20 \mathrm{~mL} \times 3)$, 合并有机相, 饱和氯化钠溶液洗, 有机相用无水硫酸钠干燥, 减压蒸除溶剂, 粗品经柱层 析纯化 [洗脱剂为 $V$ (石油醚) : $V$ (乙酸乙酯 $)=30: 1]^{[23]}$.

3-苯基丙烯酸乙酯(3a): $7.93 \mathrm{~g}$, 黄色油状物, 收率 90\%. ${ }^{1} \mathrm{H}$ NMR (DMSO- $\left.d_{6}, 400 \mathrm{MHz}\right) \delta: 1.28(\mathrm{t}, J=7.2$ $\mathrm{Hz}, 3 \mathrm{H}, \mathrm{OCH}_{2} \mathrm{CH}_{3}$ ), 4.22 (q, J=7.2 Hz, 2H, $\mathrm{OCH}_{2}$ ), 6.66 $(\mathrm{d}, J=16.0 \mathrm{~Hz}, 1 \mathrm{H},=\mathrm{CHCOOEt}), 7.42 \sim 7.44(\mathrm{~m}, 3 \mathrm{H}$, $\operatorname{ArH}), 7.66(\mathrm{~d}, J=16.0 \mathrm{~Hz}, 1 \mathrm{H}, \mathrm{ArCH}=), 7.70 \sim 7.72(\mathrm{~m}$, $2 \mathrm{H}, \mathrm{ArH})$.

3-(3,4-二甲氧基苯基)-丙烯酸乙酯(以 3,4-二甲氧基 苯甲醛为原料)(3b): $10.78 \mathrm{~g}$, 淡黄色粉末，收率 $91.3 \%$. m.p. $60 \sim 62{ }^{\circ} \mathrm{C} ;{ }^{1} \mathrm{H}$ NMR (DMSO- $\left.d_{6}, 400 \mathrm{MHz}\right) \delta: 1.25$ (t, $\left.J=7.2 \mathrm{~Hz}, 3 \mathrm{H}, \mathrm{OCH}_{2} \mathrm{CH}_{3}\right), 3.79\left(\mathrm{~s}, 3 \mathrm{H}, \mathrm{OCH}_{3}\right), 3.80$ (s, $3 \mathrm{H}, \mathrm{OCH}_{3}$ ), 4.18 (q, $\left.J=7.2 \mathrm{~Hz}, 2 \mathrm{H}, \mathrm{OCH}_{2}\right), 6.57$ (d, $J=$ $16.0 \mathrm{~Hz}, 1 \mathrm{H},=\mathrm{CHCOOEt}), 6.99(\mathrm{~d}, J=8.0 \mathrm{~Hz}, 1 \mathrm{H}$, $\operatorname{ArH}), 7.25$ (d, J=8.0 Hz, 1H, ArH), 7.36 (s, 1H, ArH), $7.60(\mathrm{~d}, J=16.0 \mathrm{~Hz}, 1 \mathrm{H}, \mathrm{ArCH}=)$.

\subsection{4 $4 \mathrm{a}$ 和 $4 \mathrm{~b}$ 的合成}

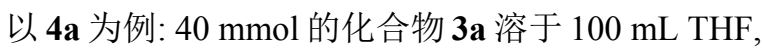
冰浴搅拌 $15 \mathrm{~min}$, 加入 $48 \mathrm{mmol}$ 的 Tosmic 和 $60 \mathrm{mmol}$ 的叔丁醇钾, 继续冰浴搅拌反应 $1 \mathrm{~h}$ 后撤冰浴, 转室温 反应. TLC 监测产物没有明显变化后, 旋干 $\mathrm{THF}$, 加水 $100 \mathrm{~mL}$, 乙酸乙酯萃取 $(25 \mathrm{~mL} \times 3)$, 合并有机相, 饱和 氯化钠溶液洗, 有机相经无水硫酸钠干燥, 减压蒸除溶 剂, 粗品经柱层析纯化 [洗脱剂为: $V$ (石油醚) : $V$ (乙酸 乙酯) $=5: 1]$.

4-苯基-1H-吡咯-3-羧酸乙酯(4a)：4.56 g, 淡黄色粉 末, 收率 53\%. m.p. 88 90 ${ }^{\circ} \mathrm{C} ;{ }^{1} \mathrm{H}$ NMR (DMSO- $d_{6}, 400$ $\mathrm{MHz}) \delta: 1.18\left(\mathrm{t}, J=7.2 \mathrm{~Hz}, 3 \mathrm{H}, \mathrm{OCH}_{2} \mathrm{CH}_{3}\right), 4.11$ (q, $J=$ $\left.7.2 \mathrm{~Hz}, 2 \mathrm{H}, \mathrm{OCH}_{2}\right), 6.93$ (s, 1H, pyrrole- H), 7.21 (t, $J=$ $7.2 \mathrm{~Hz}, 1 \mathrm{H}, \mathrm{ArH}), 7.30$ (t, J=7.2 Hz, 2H, ArH), 7.44 (d, $J=7.2 \mathrm{~Hz}, 2 \mathrm{H}, \mathrm{ArH}), 7.47$ (s, 1H, pyrrole- H), 11.53 (s, $1 \mathrm{H}$, pyrrole-NH).

4-(3,4-二甲氧基苯基)- $1 H$-吡咯-3-羧酸乙酯(以 $\mathbf{3 b}$ 为 原料)(4b): $6.16 \mathrm{~g}$, 白色粉末, 收率 56\%. m.p. 149 $151{ }^{\circ} \mathrm{C} ;{ }^{1} \mathrm{H}$ NMR (DMSO- $\left.d_{6}, 400 \mathrm{MHz}\right) \delta: 1.19$ (t, $J=7.2$ $\left.\mathrm{Hz}, 3 \mathrm{H}, \mathrm{OCH}_{2} \mathrm{CH}_{3}\right), 3.75\left(\mathrm{~s}, 6 \mathrm{H}, \mathrm{OCH}_{3}\right), 4.12$ (q, $J=7.2$ $\left.\mathrm{Hz}, 2 \mathrm{H}, \mathrm{OCH}_{2}\right), 6.87 \sim 6.91(\mathrm{~m}, 2 \mathrm{H}$, ArH, pyrrole-H), 6.98 (d, $J=8.0 \mathrm{~Hz}, 1 \mathrm{H}, \mathrm{ArH}), 7.08$ (s, 1H, pyrrole-H), 7.44 (t, $J=2.8 \mathrm{~Hz}, 1 \mathrm{H}, \mathrm{ArH}), 11.48$ (s, 1H, pyrrole-NH).

\section{$3.2 .55 \mathbf{a}$ 和 $5 \mathbf{b}$ 的合成}

以 $5 \mathbf{a}$ 为例: $20 \mathrm{mmol}$ 的化合物 $\mathbf{4 a}$ 溶于 $50 \mathrm{~mL}$ 乙醇, 搅拌, 滴加 $100 \mathrm{mmol}$ 的 $\mathrm{NaOH}(4 \mathrm{~mol} / \mathrm{L})$ 溶液, 加毕, 升 温至回流反应, TLC 监测反应结束后蒸除乙醇, 加水 20 $\mathrm{mL}$, 乙酸乙酯 $(10 \mathrm{~mL} \times 3)$ 洗, 冰浴, 水相中滴加浓盐酸 调节 $\mathrm{pH}$ 至 $2 \sim 3$, 出现大量沉淀, 抽滤, 滤饼重结晶 ${ }^{[24]}$.

4-苯基-1H-吡咯-3-羧酸(5a)：3.22 g, 淡黄色固体, 收率 86\%. m.p. 175 177 ${ }^{\circ} \mathrm{C} ;{ }^{1} \mathrm{H}$ NMR (DMSO- $d_{6}, 400$ $\mathrm{MHz}) \delta: 6.91$ (t, $J=2.4 \mathrm{~Hz}, 1 \mathrm{H}$, pyrrole-H), 7.19 (t, $J=7.2$ $\mathrm{Hz}, 1 \mathrm{H}, \mathrm{ArH}), 7.29$ (t, J=7.2 Hz, 2H, ArH), 7.43 (t, $J=$ $2.4 \mathrm{~Hz}, 1 \mathrm{H}$, pyrrole-H), 7.47 (d, J=7.2 Hz, 2H, ArH), 11.45 (s, 1H, COOH), 11.55 (s, 1H, pyrrole-NH).

4-(3,4-二甲氧基苯基)- $1 H$-吡咯-3-羧酸(以 $\mathbf{4 b}$ 为原 料)(5b): $4.05 \mathrm{~g}$, 淡黄色固体, 收率 82\%. m.p. 205 $207{ }^{\circ} \mathrm{C} ;{ }^{1} \mathrm{H}$ NMR (DMSO-d $\left.6,400 \mathrm{MHz}\right) \delta$ : 3.74 (s, 3H, 
$\mathrm{OMe}), 3.75$ (s, 3H, OMe), 6.86 6.89 (m, 2H, ArH, pyrrole- H), $7.00(\mathrm{~d}, J=8.0 \mathrm{~Hz}, 1 \mathrm{H}, \mathrm{ArH}), 7.13(\mathrm{~s}, 1 \mathrm{H}, \mathrm{ArH})$, 7.41 (t, $J=2.4 \mathrm{~Hz}, 1 \mathrm{H}$, pyrrole-H), 11.39 (s, 1H, $\mathrm{COOH})$, 11.52 (s, 1H, pyrrole-NH).

\subsection{6 $6 \mathrm{a} \sim 6 \mathrm{p}$ 的合成通法}

取 $2 \mathrm{mmol}$ 的 $\mathbf{5 a}$ (或 $\mathbf{5 b}$ ) 溶于 $30 \mathrm{~mL}$ 二氯甲烷，冰浴， 加入 $2.4 \mathrm{mmol}$ 的 HOBT 及 $2.4 \mathrm{mmol}$ 用三乙胺 $(3.6 \mathrm{mmol})$ 处理过的 EDCI. 加完搅拌活化 $30 \mathrm{~min}$ 后, 加入 2.6 $\mathrm{mmol}$ 先前制备的氨基酸甲酯盐酸盐(加入之前用 3.4 $\mathrm{mmol}$ 三乙胺处理), 反应 4 $24 \mathrm{~h}$. TLC 监测反应完成后, 反应液先水洗 $(5 \mathrm{~mL} \times 2)$, 饱和碳酸氢钠溶液洗, 饱和食 盐水洗, 有机相经无水硫酸钠干燥, 减压蒸干溶剂得粗 品, 粗品经柱硅胶层析纯化 [洗脱剂为 $V$ (石油醚)： $V$ (乙 酸乙酯) $=1: 1 \sim 1: 2]$, 得化合物 $\mathbf{6 a} \sim 6 \mathbf{6} \mathbf{p}^{[25]}$, 收率 $65 \% \sim 95 \%$.

\subsection{7 $7 \mathbf{a} \sim 7 \mathbf{p}$ 的合成通法}

取 $1 \mathrm{mmol}$ 的 $\mathbf{6 a} \sim \mathbf{6 p}$ 溶解于 $10 \mathrm{~mL} \mathrm{THF}$ 中, 加入 4 $\mathrm{mmol} \mathrm{NaOH}$ 溶液 $(1 \mathrm{~mol} / \mathrm{L})$, 搅拌, 反应. TLC 监测反应 结束后旋干 $\mathrm{THF}$, 加入 $10 \mathrm{~mL}$ 水, 乙酸乙酯洗 3 次, 有 机层弃掉, 冰浴条件下, 水相滴加 $1 \mathrm{~mol} / \mathrm{L}$ 的盐酸溶液 调节 $\mathrm{pH}$ 至 $2 \sim 3$, 有大量沉淀析出, 乙酸乙酯萃取 (15 $\mathrm{mL} \times 3)$, 合并有机相, 无水硫酸钠干燥, 抽滤, 旋干溶 剂, 粗品重结晶, 得到化合物 $7 \mathbf{a} \sim 7 \mathbf{p}$.

$N$-(4-苯基- $1 H$-吡咯-3-羰基)- $L$-甘氨酸 (7a): 淡黄色 固体, 收率 83\%. m.p. 205 207 ${ }^{\circ} \mathrm{C} ;{ }^{1} \mathrm{H}$ NMR (DMSO- $d_{6}$, $400 \mathrm{MHz}) \delta: 3.81$ (d, $\left.J=6.0 \mathrm{~Hz}, 2 \mathrm{H}, \mathrm{NCH}_{2} \mathrm{COO}\right), 6.93(\mathrm{t}$, $J=2.4 \mathrm{~Hz}, 1 \mathrm{H}$, pyrrole- $\mathrm{H}), 7.15(\mathrm{t}, J=7.2 \mathrm{~Hz}, 1 \mathrm{H}, \mathrm{ArH})$, 7.27 (t, $J=7.2 \mathrm{~Hz}, 2 \mathrm{H}, \mathrm{ArH}), 7.29$ (t, $J=2.4 \mathrm{~Hz}, 1 \mathrm{H}$, pyrrole-H), 7.47 (d, $J=7.2 \mathrm{~Hz}, 2 \mathrm{H}, \mathrm{ArH}), 7.90(\mathrm{t}, J=6.0 \mathrm{~Hz}$, $1 \mathrm{H}, \mathrm{CONH}), 11.24(\mathrm{~s}, 1 \mathrm{H}$, pyrrole-NH), $12.40(\mathrm{~s}, 1 \mathrm{H}$, $\mathrm{COOH}) ;{ }^{13} \mathrm{C}$ NMR (100 MHz, DMSO- $\left.d_{6}\right) \delta: 172.3,165.8$, 136.0, 128.8 (2C), 128.1 (2C), 125.9, 124.3, 122.1, 118.7, 117.1, 41.3; ESI-HRMS calcd for $\mathrm{C}_{13} \mathrm{H}_{13} \mathrm{~N}_{2} \mathrm{O}_{3}[\mathrm{M}+\mathrm{H}]^{+}$ 245.0926, found 245.0916.

$N$-(4-苯基- $1 H$-吡咯-3-羰基)- $L$-丙氨酸(7b): 淡黄色 固体, 收率 85\%. m.p. $165 \sim 167{ }^{\circ} \mathrm{C} ;{ }^{1} \mathrm{H}$ NMR (DMSO- $d_{6}$, $300 \mathrm{MHz}) \delta: 1.31$ (d, $\left.J=7.2 \mathrm{~Hz}, 3 \mathrm{H}, \mathrm{NCCH}_{3}\right), 4.24 \sim 4.34$ (m, 1H, NCH), $6.93(\mathrm{t}, J=2.4 \mathrm{~Hz}, 1 \mathrm{H}$, pyrrole-H), $7.18(\mathrm{t}$, $J=7.2 \mathrm{~Hz}, 1 \mathrm{H}, \mathrm{ArH}), 7.26(\mathrm{t}, J=7.2 \mathrm{~Hz}, 2 \mathrm{H}, \mathrm{ArH}), 7.31(\mathrm{t}$, $J=2.4 \mathrm{~Hz}, 1 \mathrm{H}$, pyrrole-H), $7.46(\mathrm{~d}, J=7.2 \mathrm{~Hz}, 2 \mathrm{H}, \mathrm{ArH})$, $7.74(\mathrm{~d}, J=7.2 \mathrm{~Hz}, 1 \mathrm{H}, \mathrm{CONH}), 11.24$ (s, $1 \mathrm{H}$, pyrrole-NH), 12.39 (br s, $1 \mathrm{H}, \mathrm{COOH}) ;{ }^{13} \mathrm{C}$ NMR $(100 \mathrm{MHz}$, DMSO- $\left.d_{6}\right) \delta: 175.1,165.4,136.0,128.8(2 \mathrm{C}), 128.2(2 \mathrm{C})$, 126.0, 124.2, 122.3, 118.5, 117.1, 48.1, 17.6; ESI-HRMS calcd for $\mathrm{C}_{14} \mathrm{H}_{15} \mathrm{~N}_{2} \mathrm{O}_{3}[\mathrm{M}+\mathrm{H}]^{+}$259.1083, found
259.1084 .

$N$-(4-苯基- $1 H$-吡咯-3-羰基)- $L$-颎氨酸(7c)：淡黄色 固体, 收率 79\%. m.p. 199 201 ${ }^{\circ} \mathrm{C} ;{ }^{1} \mathrm{H}$ NMR (DMSO- $d_{6}$, $400 \mathrm{MHz}) \delta: 0.80$ (d, $\left.J=6.8 \mathrm{~Hz}, 3 \mathrm{H}, \mathrm{CH}_{3}\right), 0.88$ (d, $J=6.8$ $\left.\mathrm{Hz}, 3 \mathrm{H}, \mathrm{CH}_{3}\right), 2.10 \sim 2.01\left(\mathrm{~m}, 1 \mathrm{H}, \mathrm{CH}\left(\mathrm{CH}_{3}\right)_{2}\right), 4.23(\mathrm{dd}$, $J=8.0,6.0 \mathrm{~Hz}, 1 \mathrm{H}, \mathrm{NCH}), 6.91(\mathrm{t}, J=2.4 \mathrm{~Hz}, 1 \mathrm{H}$, pyrroleH), $7.11(\mathrm{~d}, J=8.0 \mathrm{~Hz}, 1 \mathrm{H}, \mathrm{CONH}), 7.20$ (t, $J=7.2 \mathrm{~Hz}$, $1 \mathrm{H}, \mathrm{ArH}), 7.30$ (t, $J=7.2 \mathrm{~Hz}, 2 \mathrm{H}, \mathrm{ArH}), 7.34$ (t, $J=2.4$ $\mathrm{Hz}, 1 \mathrm{H}$, pyrrole-H), 7.44 (d, J=7.2 Hz, 1H, ArH), 11.29 (s, 1H, pyrrole-NH), 12.48 (brs, $1 \mathrm{H}, \mathrm{COOH}$ ); ${ }^{13} \mathrm{C}$ NMR $\left(100 \mathrm{MHz}, \mathrm{DMSO}-d_{6}\right) \delta: 173.8,165.5,136.0,129.0$ (2C), 128.4 (2C), 126.3, 123.8, 122.8, 118.6, 117.2, 57.8, 30.1, 19.7, 18,6; ESI-HRMS calcd for $\mathrm{C}_{16} \mathrm{H}_{19} \mathrm{~N}_{2} \mathrm{O}_{3}[\mathrm{M}+\mathrm{H}]^{+}$ 287.1396, found 287.1394.

$N$-(4-苯基- $1 H$-吡咯-3-羰基)- $L$-亮氨酸(7d)：淡黄色 固体, 收率 78\%. m.p. $184 \sim 186{ }^{\circ} \mathrm{C} ;{ }^{1} \mathrm{H}$ NMR (DMSO- $d_{6}$, $400 \mathrm{MHz}) \delta: 0.86 \sim 0.90\left(\mathrm{~m}, 6 \mathrm{H}, \mathrm{C}\left(\mathrm{CH}_{3}\right)_{2}\right), 1.41 \sim 1.73(\mathrm{~m}$, $\left.3 \mathrm{H}, \mathrm{CH}_{2} \mathrm{CH}\right), 4.27 \sim 4.39(\mathrm{~m}, 1 \mathrm{H}, \mathrm{NCH}), 6.93(\mathrm{~s}, 1 \mathrm{H}$, pyrrole-H), 7.17 (t, $J=7.2 \mathrm{~Hz}, 1 \mathrm{H}, \mathrm{ArH}), 7.26$ (t, $J=7.2 \mathrm{~Hz}$, 2H, ArH), 7.31 (s, $1 \mathrm{H}$, pyrrole-H), 7.45 (d, $J=7.2 \mathrm{~Hz}, 2 \mathrm{H}$, ArH), 7.63 (d, $J=7.6 \mathrm{~Hz}, 1 \mathrm{H}, \mathrm{CONH}), 11.25$ (s, 1H, pyrrole-NH), 12.43 (brs, $1 \mathrm{H}, \mathrm{COOH}) ;{ }^{13} \mathrm{C}$ NMR $(100 \mathrm{MHz}$, DMSO- $\left.d_{6}\right) \delta: 175.1,165.6,136.0,128.8(2 \mathrm{C}), 128.2(2 \mathrm{C})$, $126.0,124.1,122.3,118.5,117.2,50.8,25.0,23.4,21.8$ (2C); ESI-HRMS calcd for $\mathrm{C}_{17} \mathrm{H}_{21} \mathrm{~N}_{2} \mathrm{O}_{3}[\mathrm{M}+\mathrm{H}]^{+}$ 301.1552, found 301.1523 .

$N$-(4-苯基- $1 H$-吡咯-3-羰基)- $L$-苯丙氨酸 (7e) : 淡黄 色固体, 收率 87\%. m.p. 94 96 ${ }^{\circ} \mathrm{C} ;{ }^{1} \mathrm{H}$ NMR (DMSO- $d_{6}$, $400 \mathrm{MHz}) \delta: 2.91 \sim 2.97(\mathrm{~m}, 1 \mathrm{H}, \mathrm{ArCH}), 3.09 \sim 3.13(\mathrm{~m}$, $1 \mathrm{H}, \mathrm{ArCH}), 4.50 \sim 4.56(\mathrm{~m}, 1 \mathrm{H}, \mathrm{NCH}), 6.90$ (t, $J=2.4 \mathrm{~Hz}$, $1 \mathrm{H}$, pyrrole- $\mathrm{H}), 7.12 \sim 7.33(\mathrm{~m}, 11 \mathrm{H}, \mathrm{ArH}$, pyrrole- $\mathrm{H})$, $7.72(\mathrm{~d}, J=8.0 \mathrm{~Hz}, 1 \mathrm{H}, \mathrm{CONH}), 11.22$ (s, $1 \mathrm{H}$, pyrrole$\mathrm{NH}), 12.62$ (brs, $1 \mathrm{H}, \mathrm{COOH}) ;{ }^{13} \mathrm{C}$ NMR $(100 \mathrm{MHz}$, DMSO- $\left.d_{6}\right) \delta: 174.0,165.3,138.5,135.8,129.6(2 \mathrm{C}), 128.7$ (2C), 128.6 (2C), 128.2 (2C), 126.8, 125.9, 124.1, 122.2, 118.6, 117.1, 53.9, 37.0; ESI-HRMS calcd for $\mathrm{C}_{20} \mathrm{H}_{19} \mathrm{~N}_{2} \mathrm{O}_{3}$ $[\mathrm{M}+\mathrm{H}]^{+}$335.1396, found 335.1385.

$N$-(4-苯基- $1 H$-吡咯-3-羰基)- $L$-脯氨酸(7f)：淡黄色 固体, 收率 65\%. m.p. 106 108 ${ }^{\circ} \mathrm{C} ;{ }^{1} \mathrm{H}$ NMR (DMSO- $d_{6}$, $400 \mathrm{MHz}) \delta: 1.56 \sim 1.89$ (m, 3H, pyrrolidine-H), $2.13 \sim$ $2.16(\mathrm{~m}, 1 \mathrm{H}$, pyrrolidine- $\mathrm{H}), 3.30 \sim 3.41(\mathrm{~m}, 2 \mathrm{H}$, pyrrolidine-H), $4.2 \sim 4.4(\mathrm{~m}, 1 \mathrm{H}$, pyrrolidine- $\mathrm{H}), 6.86 \sim 7.09(\mathrm{~m}$, 2H, pyrrole-H), 7.13 (t, $J=7.2 \mathrm{~Hz}, 1 \mathrm{H}, \mathrm{ArH}), 7.25$ (t, $J=$ $7.2 \mathrm{~Hz}, 2 \mathrm{H}, \mathrm{ArH}), 7.46$ (d, J=7.2 Hz, 2H, ArH), 11.24 (s, $1 \mathrm{H}$, pyrrole-NH), 12.45 (brs, $1 \mathrm{H}, \mathrm{COOH}) ;{ }^{13} \mathrm{C}$ NMR (100 
MHz, DMSO- $\left.d_{6}\right) \delta: 173.7,165.9,135.4,128.1$ (2C), 126.6 (2C), 125.2, 122.6, 120.0, 117.0, 116.7, 58.5, 48.5, 29.1, 24.5; ESI-HRMS calcd for $\mathrm{C}_{16} \mathrm{H}_{17} \mathrm{~N}_{2} \mathrm{O}_{3}[\mathrm{M}+\mathrm{H}]^{+}$ 285.1239, found 285.1235.

$N$-(4-苯基- $1 H$-吡咯-3-羰基)- $L$-丝氨酸(7g): 黄色固 体, 收率 80\%. m.p. 174 176 ${ }^{\circ} \mathrm{C} ;{ }^{1} \mathrm{H}$ NMR (DMSO- $d_{6}$, $400 \mathrm{MHz}) \delta: 3.66 \sim 3.75\left(\mathrm{~m}, 2 \mathrm{H}, \mathrm{CCH}_{2}\right), 4.34 \sim 4.39(\mathrm{~m}$, $1 \mathrm{H}, \mathrm{NCH}), 4.90$ (brs, $1 \mathrm{H}, \mathrm{OH}), 6.93$ (t, $J=2.4 \mathrm{~Hz}, 1 \mathrm{H}$, pyrrole-H), 7.17 (d, $J=7.2 \mathrm{~Hz}, 1 \mathrm{H}, \mathrm{ArH}), 7.27$ (t, $J=7.2$ $\mathrm{Hz}, 2 \mathrm{H}, \mathrm{ArH}), 7.35$ (t, $J=2.4 \mathrm{~Hz}, 1 \mathrm{H}$, pyrrole-H), 7.41 (d, $J=7.6 \mathrm{~Hz}, 1 \mathrm{H}, \mathrm{CONH}), 7.47(\mathrm{~d}, J=7.2 \mathrm{~Hz}, 2 \mathrm{H}, \mathrm{ArH})$, 11.29 (s, $1 \mathrm{H}$, pyrrole-NH), 12.52 (brs, $1 \mathrm{H}, \mathrm{COOH}) ;{ }^{13} \mathrm{C}$ NMR (100 MHz, DMSO- $\left.d_{6}\right) \delta$ : 172.8, 165.3, 135.8, 128.8 (2C), 128.3 (2C), 126.0, 124.1, 122.6, 118.7, 117.1, 61.9, 55.3; ESI-HRMS calcd for $\mathrm{C}_{14} \mathrm{H}_{15} \mathrm{~N}_{2} \mathrm{O}_{4}[\mathrm{M}+\mathrm{H}]{ }^{+}$ 275.1032, found 275.1024 .

$N$-(4-苯基- $1 H$-吡咯-3-羰基)- $L$-天冬氨酸(7h): 灰白 色固体, 收率 89\%, m.p. $181 \sim 183{ }^{\circ} \mathrm{C} ;{ }^{1} \mathrm{H}$ NMR $\left(\mathrm{DMSO}_{6}, 400 \mathrm{MHz}\right) \delta: 2.60 \sim 2.66(\mathrm{~m}, 1 \mathrm{H}, \mathrm{CCH})$, $2.72 \sim 2.78(\mathrm{~m}, 1 \mathrm{H}, \mathrm{CCH}), 4.65$ (q, $J=7.2 \mathrm{~Hz}, 1 \mathrm{H}, \mathrm{NCH})$, $6.93(\mathrm{t}, J=2.0 \mathrm{~Hz}, 1 \mathrm{H}$, pyrrole-H), $7.16(\mathrm{t}, J=7.2 \mathrm{~Hz}, 1 \mathrm{H}$, ArH), 7.26 (t, $J=7.2 \mathrm{~Hz}, 2 \mathrm{H}, \mathrm{ArH}), 7.28(\mathrm{t}, J=2.0 \mathrm{~Hz}$, $1 \mathrm{H}$, pyrrole-H), 7.45 (d, $J=7.2 \mathrm{~Hz}, 2 \mathrm{H}, \mathrm{ArH}), 7.76$ (d, $J=$ $8.0 \mathrm{~Hz}, 1 \mathrm{H}, \mathrm{CONH}), 11.29$ (s, 1H, pyrrole-NH), 12.46 (brs, $2 \mathrm{H}, \mathrm{COOH}) ;{ }^{13} \mathrm{C}$ NMR (100 MHz, DMSO- $\left.d_{6}\right) \delta: 173.3$, 172.4, 165.2, 135.8, 128.8 (2C), 128.2 (2C), 126.0, 124.1, 122.5, 118.7, 117.0, 49.2, 36.5; ESI-HRMS calcd for $\mathrm{C}_{15} \mathrm{H}_{15} \mathrm{~N}_{2} \mathrm{O}_{5}[\mathrm{M}+\mathrm{H}]^{+}$303.0981, found 303.0977.

$N$-[4-(3,4-二甲氧基)-苯基- $1 H$-吡咯-3-羰基]- $L$-甘氨 酸(7i): 淡黄色固体, 收率 $85 \% .201 \sim 203{ }^{\circ} \mathrm{C} ;{ }^{1} \mathrm{H}$ NMR (DMSO- $\left.d_{6}, 400 \mathrm{MHz}\right) \delta: 3.733\left(\mathrm{~s}, 3 \mathrm{H}, \mathrm{OCH}_{3}\right), 3.737$ (s, $\left.1 \mathrm{H}, \mathrm{OCH}_{3}\right), 3.78\left(\mathrm{~d}, J=5.6 \mathrm{~Hz}, 2 \mathrm{H}, \mathrm{NCH}_{2}\right), 6.87$ (d, $J=$ $8.4 \mathrm{~Hz}, 1 \mathrm{H}, \mathrm{ArH}), 6.88$ (t, $J=2.4 \mathrm{~Hz}, 1 \mathrm{H}$, pyrrole-H), 7.00 (d, $J=8.4 \mathrm{~Hz}, 1 \mathrm{H}, \mathrm{ArH}), 7.10$ (s, 1H, ArH), 7.25 (t, $J=2.4$ Hz, 1H, pyrrole-H), 7.63 (brs, 1H, CONH), 11.21 (s, 1H, pyrrole-NH); ${ }^{13} \mathrm{C}$ NMR (100 MHz, DMSO- $\left.d_{6}\right) \delta: 172.5$, $165.8,148.6,147.5,128.7,123.9,122.1,120.7,118.2$, 117.2, 113.2, 112.1, 56.0, 55.8, 41.7; ESI-HRMS calcd for $\mathrm{C}_{15} \mathrm{H}_{17} \mathrm{~N}_{2} \mathrm{O}_{5}[\mathrm{M}+\mathrm{H}]^{+}$305.1137, found 305.1126.

$N$-[4-(3,4-二甲氧基)-苯基- $1 H$-吡咯-3-羰基]- $L$-丙氨 酸(7j): 淡黄色固体, 收率 $80 \%$. m.p. $85 \sim 87{ }^{\circ} \mathrm{C} ;{ }^{1} \mathrm{H}$ NMR (DMSO- $\left.d_{6}, 300 \mathrm{MHz}\right) \delta$ : $1.29(\mathrm{~d}, J=7.2 \mathrm{~Hz}, 3 \mathrm{H}$, $\mathrm{CH}_{3}$ ), 3.727 (s, $\left.3 \mathrm{H}, \mathrm{OCH}_{3}\right), 3.735\left(\mathrm{~s}, 3 \mathrm{H}, \mathrm{OCH}_{3}\right), 4.23 \sim$ $4.32(\mathrm{~m}, 1 \mathrm{H}, \mathrm{NCH}), 6.84 \sim 6.87(\mathrm{~m}, 2 \mathrm{H}, \mathrm{ArH}$, pyrrole-H), 6.98 (d, J=8.4 Hz, 1H, ArH), 7.07 (s, 1H, ArH), 7.25 (brs,
1H, pyrrole-H), 7.58 (d, $J=7.5 \mathrm{~Hz}, 1 \mathrm{H}, \mathrm{CONH}), 11.20$ (s, $1 \mathrm{H}$, pyrrole-NH), 12.35 (brs, $1 \mathrm{H}, \mathrm{COOH}) ;{ }^{13} \mathrm{C}$ NMR $(100$ MHz, DMSO- $\left.d_{6}\right) \delta: 174.5,164.9,148.1,147.1,128.2$, 123.4, 121.8, 120.3, 117.6, 116.6, 112.8, 111.6, 55.6, 55.3, 47.6, 17.2; ESI-HRMS calcd for $\mathrm{C}_{16} \mathrm{H}_{19} \mathrm{~N}_{2} \mathrm{O}_{5}[\mathrm{M}+\mathrm{H}]^{+}$ 319.1294, found 319.1278 .

$N$-[4-(3,4-二甲氧基)-苯基- $1 H$-吡咯-3-羰基]- $L$-坃氨 酸 $(7 \mathbf{k})$ : 淡黄色固体, 收率 $87 \%$. m.p. $89 \sim 91{ }^{\circ} \mathrm{C} ;{ }^{1} \mathrm{H}$ NMR (DMSO- $\left.d_{6}, 400 \mathrm{MHz}\right) \delta: 0.73(\mathrm{~d}, J=6.8 \mathrm{~Hz}, 3 \mathrm{H}$, $\left.\mathrm{CH}_{3}\right), 0.84\left(\mathrm{~d}, J=6.8 \mathrm{~Hz}, 3 \mathrm{H}, \mathrm{CH}_{3}\right), 1.98 \sim 2.08(\mathrm{~m}, 1 \mathrm{H}$, $\left.\mathrm{CH}\left(\mathrm{CH}_{3}\right)_{2}\right), 3.74\left(\mathrm{~s}, 6 \mathrm{H}, \mathrm{OCH}_{3}\right), 4.20 \sim 4.23(\mathrm{~m}, 1 \mathrm{H}$, $\mathrm{NCH}), 6.85$ (d, $J=2.4 \mathrm{~Hz}, 1 \mathrm{H}$, pyrrole-H), $6.89 \sim 6.96(\mathrm{~m}$, $3 \mathrm{H}, \mathrm{ArH}, \mathrm{CONH}), 7.02$ (d, $J=2.0 \mathrm{~Hz}, 1 \mathrm{H}, \mathrm{ArH}), 7.29$ (t, $J=2.4 \mathrm{~Hz}, 1 \mathrm{H}$, pyrrole-H), 11.25 (s, 1H, pyrrole-NH), 12.36 (brs, $1 \mathrm{H}, \mathrm{COOH}) ;{ }^{13} \mathrm{C}$ NMR (100 MHz, DMSO- $d_{6}$ ) $\delta: 173.3,164.9,148.4,147.4,128.1,123.0,122.2,120.7$, $117.8,116.8,112.9,111.9,57.1,55.6,55.3,29.6,19.2$, 17.8; ESI-HRMS calcd for $\mathrm{C}_{18} \mathrm{H}_{23} \mathrm{~N}_{2} \mathrm{O}_{5}[\mathrm{M}+\mathrm{H}]{ }^{+}$ 347.1607 , found 347.1595 .

$N$-[4-(3,4-二甲氧基)-苯基- $1 H$-吡咯-3-羰基]- $L$-亮氨 酸(71): 淡黄色固体, 收率 $84 \%$. m.p. $87 \sim 89{ }^{\circ} \mathrm{C} ;{ }^{1} \mathrm{H}$ NMR (DMSO- $\left.d_{6}, 400 \mathrm{MHz}\right) \delta: 0.86(\mathrm{t}, J=6.8 \mathrm{~Hz}, 6 \mathrm{H}$, $\left.\mathrm{CH}_{3}\right), 1.48 \sim 1.61\left(\mathrm{~m}, 3 \mathrm{H}, \mathrm{CH}_{2} \mathrm{CH}\right), 3.73\left(\mathrm{~s}, 3 \mathrm{H}, \mathrm{OCH}_{3}\right)$, $3.74\left(\mathrm{~s}, 3 \mathrm{H}, \mathrm{OCH}_{3}\right), 4.29 \sim 4.34(\mathrm{~m}, 1 \mathrm{H}, \mathrm{NCH}), 6.86 \sim 6.88$ (m, $2 \mathrm{H}$, ArH, pyrrole-H), 6.97 (d, $J=8.0 \mathrm{~Hz}, 1 \mathrm{H}, \mathrm{ArH})$, 7.06 (s, 1H, ArH), 7.25 (brs, 1H, pyrrole-H), 7.32 (d, $J=$ $8.0 \mathrm{~Hz}, 1 \mathrm{H}, \mathrm{CONH}) 11.15$ (s, 1H, pyrrole-NH), 12.32 (brs, $1 \mathrm{H}, \mathrm{COOH}) ;{ }^{13} \mathrm{C}$ NMR $\left(100 \mathrm{MHz}, \mathrm{DMSO}-d_{6}\right) \delta: 175.1$, $165.6,148.6,147.6,128.7,123.9,122.2,120.7,118.1$, 117.2, 113.2, 112.1, 56.0, 55.8, 50.7, 24.9, 23.4, 21.8; ESI-HRMS calcd for $\mathrm{C}_{19} \mathrm{H}_{25} \mathrm{~N}_{2} \mathrm{O}_{5}[\mathrm{M}+\mathrm{H}]^{+} 361.1763$, found 361.1759 .

$N$-[4-(3,4-二甲氧基)-苯基- $1 H$-吡咯-3-羰基]- $L$-苯丙 氨酸 $(7 \mathrm{~m})$ : 淡黄色固体, 收率 $83 \%$. m.p. $176 \sim 178{ }^{\circ} \mathrm{C}$; ${ }^{1} \mathrm{H}$ NMR (DMSO- $\left.d_{6}, 400 \mathrm{MHz}\right) \delta: 2.91 \sim 2.97(\mathrm{~m}, 1 \mathrm{H}$, $\mathrm{ArCH}), 3.10 \sim 3.15(\mathrm{~m}, 1 \mathrm{H}, \mathrm{ArCH}), 3.68\left(\mathrm{~s}, 3 \mathrm{H}, \mathrm{OCH}_{3}\right)$, $3.73\left(\mathrm{~s}, 3 \mathrm{H}, \mathrm{OCH}_{3}\right), 4.43 \sim 4.48(\mathrm{~m}, 1 \mathrm{H}, \mathrm{NCH}), 6.80(\mathrm{~d}$, $J=8.0 \mathrm{~Hz}, 1 \mathrm{H}, \mathrm{ArH}), 6.83 \sim 6.86(\mathrm{~m}, 2 \mathrm{H}, \mathrm{ArH}$, pyrrole-H), 7.01 (s, 1H, ArH), $7.13 \sim 7.25$ (m, 8H, ArH, pyrrole-H, CONH), 11.21(s, $1 \mathrm{H}$, pyrrole-NH); ${ }^{13} \mathrm{C}$ NMR (100 MHz, DMSO- $\left.d_{6}\right) \delta: 175.0,165.1,148.6,147.6,140.0$, 129.7 (2C), 128.6, 128.4 (2C), 126.5, 123.6, 122.1, 120.8, $118.1, \quad 117.5, \quad 113.1, \quad 112.1, \quad 56.0, \quad 55.8, \quad 54.6, \quad 37.5$; ESI-HRMS calcd for $\mathrm{C}_{22} \mathrm{H}_{23} \mathrm{~N}_{2} \mathrm{O}_{5}[\mathrm{M}+\mathrm{H}]^{+}$395.1607, found 395.1588 . 
$N$-[4-(3,4-二甲氧基)-苯基- $1 H$-吡咯-3-羰基]- $L$-脯氨 酸(7n): 淡黄色固体, 收率 75\%. m.p. $230 \sim 232{ }^{\circ} \mathrm{C} ;{ }^{1} \mathrm{H}$ NMR (DMSO- $\left.d_{6}, 400 \mathrm{MHz}\right) \delta: 1.67 \sim 1.81(\mathrm{~m}, 3 \mathrm{H}$, pyrrolidine-H), $2.12 \sim 2.16(\mathrm{~m}, 1 \mathrm{H}$, pyrrolidine- $\mathrm{H}), 3.31 \sim$ $3.36(\mathrm{~m}, 2 \mathrm{H}$, pyrrolidine- $\mathrm{H}), 3.73\left(\mathrm{~s}, 3 \mathrm{H}, \mathrm{OCH}_{3}\right), 3.75(\mathrm{~s}$, $\left.3 \mathrm{H}, \mathrm{OCH}_{3}\right), 4.29 \sim 4.34(\mathrm{~m}, 1 \mathrm{H}$, pyrrolidine- $\mathrm{H}), 6.82 \sim$ $7.03(\mathrm{~m}, 5 \mathrm{H}, \mathrm{ArH}$, pyrrole-H), 11.20 (s, 1H, pyrrole- $\mathrm{NH}$ ), 12.43 (brs, $1 \mathrm{H}, \mathrm{COOH}) ;{ }^{13} \mathrm{C}$ NMR (100 MHz, DMSO- $d_{6}$ ) $\delta: 174.1,166.6,148.9,147.3,129.0,123.2,120.4,119.3$, $117.3,116.8,112.3,111.4,59.0,56.0,55.8,49.0,29.6$, 25.0; ESI-HRMS calcd for $\mathrm{C}_{18} \mathrm{H}_{21} \mathrm{~N}_{2} \mathrm{O}_{5}[\mathrm{M}+\mathrm{H}]^{+}$ 345.1450 , found 345.1439 .

$N$-[4-(3,4-二甲氧基)-苯基- $1 H$-吡咯-3-羰基] $-L$ - 丝氨 酸(7o): 黄色固体, 收率 $80 \%$. m.p. $187 \sim 189{ }^{\circ} \mathrm{C} ;{ }^{1} \mathrm{H}$ NMR (DMSO- $\left.d_{6}, 400 \mathrm{MHz}\right) \delta: 3.63 \sim 3.67(\mathrm{~m}, 1 \mathrm{H}, \mathrm{CCH})$, $3.70 \sim 3.72(\mathrm{~m}, 1 \mathrm{H}, \mathrm{CCH}), 3.73\left(\mathrm{~s}, 3 \mathrm{H}, \mathrm{OCH}_{3}\right), 3.74(\mathrm{~s}$, $\left.3 \mathrm{H}, \mathrm{OCH}_{3}\right), 4.34 \sim 4.38(\mathrm{~m}, 1 \mathrm{H}, \mathrm{NCH}), 4.90$ (brs, $1 \mathrm{H}$, $\mathrm{OH}), 6.86 \sim 6.88(\mathrm{~m}, 2 \mathrm{H}$, ArH, pyrrole- $\mathrm{H}), 6.98(\mathrm{~d}, J=8.0$ $\mathrm{Hz}, 1 \mathrm{H}, \mathrm{ArH}$ ), 7.07 (s, 1H, ArH), 7.26 (d, $J=8.0 \mathrm{~Hz}, 1 \mathrm{H}$, CONH), 7.31 (t, $J=2.4 \mathrm{~Hz}, 1 \mathrm{H}$, pyrrole-H), 11.24 (s, $1 \mathrm{H}$, pyrrole-NH), 12.52 (brs, $1 \mathrm{H}, \mathrm{COOH}) ;{ }^{13} \mathrm{C}$ NMR (100 MHz, DMSO- $\left.d_{6}\right) \delta$ : $172.8,165.3,148.7,147.7,128.5$, 123.8, 122.7, 120.8, 118.4, 117.1, 113.2, 112.2, 61.9, 56.0, 55.8, 55.2; ESI-HRMS calcd for $\mathrm{C}_{16} \mathrm{H}_{19} \mathrm{~N}_{2} \mathrm{O}_{6}[\mathrm{M}+\mathrm{H}]^{+}$ 335.1243 , found 335.1220 .

$N$-[4-(3,4-二甲氧基)-苯基- $1 H$-吡咯-3-羰基]- $L$-天冬 氨酸 (7p): 淡黄色固体, 收率 89\%. m.p. $124 \sim 126{ }^{\circ} \mathrm{C} ;{ }^{1} \mathrm{H}$ NMR (DMSO- $\left.d_{6}, 400 \mathrm{MHz}\right) \delta: 2.61 \sim 2.67(\mathrm{~m}, 1 \mathrm{H}, \mathrm{CCH})$, $2.72 \sim 2.77(\mathrm{~m}, 1 \mathrm{H}, \mathrm{CCH}), 3.74\left(\mathrm{~s}, 6 \mathrm{H}, \mathrm{OCH}_{3}\right), 4.67$ (q, $J=7.2 \mathrm{~Hz}, 1 \mathrm{H}, \mathrm{NCH}), 6.87$ (d, $J=8.0 \mathrm{~Hz}, 1 \mathrm{H}, \mathrm{ArH}), 6.88$ (t, $J=2.4 \mathrm{~Hz}, 1 \mathrm{H}$, pyrrole), 6.97 (d, $J=8.0 \mathrm{~Hz}, 1 \mathrm{H}, \mathrm{ArH}$ ), 7.06 (s, 1H, ArH), 7.24 (t, $J=2.4 \mathrm{~Hz}, 1 \mathrm{H}$, pyrrole-H), 7.64 (d, $J=8.0 \mathrm{~Hz}, 1 \mathrm{H}, \mathrm{CONH}), 11.21$ (s, $1 \mathrm{H}$, pyrrole-NH), 12.35 (brs, $2 \mathrm{H}, \mathrm{COOH}, \mathrm{COOH}) ;{ }^{13} \mathrm{C}$ NMR $(100 \mathrm{MHz}$, DMSO- $\left.d_{6}\right) \delta: 173.3,172.5,165.3,148.6,147.6,128.5$, 123.9, 122.4, 120.7, 118.2, 116.9, 113.1, 112.1, 56.0, 55.8, 49.1, 36.5; ESI-HRMS calcd for $\mathrm{C}_{17} \mathrm{H}_{19} \mathrm{~N}_{2} \mathrm{O}_{7}[\mathrm{M}+\mathrm{H}]^{+}$ 363.1192 , found 363.1180 .

\section{3 细胞增殖抑制实验}

本实验用噻唑蓝(MTT)法测定目标化合物的细胞增 殖抑制活性 ${ }^{[26]}$. MTT 法是一种检测细胞存活和生长的 常规方法, 其原理是活细胞线粒体中的琥珀酸脱氢酶能 使外源性 MTT 还原为不溶于水的蓝紫色结晶甲瓒并沉 积在细胞内, 死细胞则没有此变化. 二甲基亚砜可以溶 解甲琨，用酶联免疫检测仪在所需波长处测定其吸光值
可间接反映活细胞数量, 在一定细胞数范围内, 甲玞形 成的数量和细胞数成正比.

实验步骤: 首先, 将冻存细胞复苏, 细胞培养、换液 和传代均按标准操作进行. 取对数生长期细胞, 配制成 细胞浓度为 $5 \times 10^{4}$ 个 $/ \mathrm{mL}$ 细胞悬液, 接种到 96 孔板, 每 孔 $90 \mu \mathrm{L}$ (约 4000 5000 个细胞/孔), 边缘孔用无菌 PBS 填充. 于 $37{ }^{\circ} \mathrm{C} 、 5 \% \mathrm{CO}_{2}$ 培养箱中培养 $24 \mathrm{~h}$ 至细胞铺板 孔底, 加入不同浓度的化合物溶液. 不同浓度阳性对照 紫杉醇的样品溶液和阴性对照溶液各 $10 \mu \mathrm{L}$, 使化合物 和阳性对照浓度分别为 $0.1,1,10,20,50,100 \mu \mathrm{mol} / \mathrm{L}$, 每个浓度设置 4 个复孔. 继续培养 $24 \mathrm{~h}$ 后, 每孔加入 20 $\mu \mathrm{L}$ MTT 溶液 $(0.5 \% \mathrm{MTT})$, 继续培养 $4 \mathrm{~h}$ 后终止培养. 吸 净 96 孔板中的上清液, 每孔加入 $100 \mu \mathrm{L}$ 二甲基亚砜, 待甲瓒充分溶解后, 酶联免疫检测仪检测 $570 \mathrm{~nm}$ 处各 孔吸光值(OD 值).

细胞增殖抑制率\%=(阴性对照组平均 $\mathrm{OD}$ 值 -

给药组平均 $\mathrm{OD}$ 值) / 阴性对照组平均 $\mathrm{OD}$ 值 $\times 100 \%$

根据 $\mathrm{IC}_{50}$ 统计软件进行计算, 得到各化合物 $\mathrm{IC}_{50}$ 值.

致谢 感谢上海交通大学分析测试中心提供的所有光 谱数据.

辅助材料(Supporting Information) 所有目标化合物 的 ${ }^{1} \mathrm{H}$ NMR, ${ }^{13} \mathrm{C}$ NMR以及ESI-HRMS谱图. 这些材料可 以免费从本刊网站(http://sioc-journal.cn/)上下载.

\section{References}

[1] Joshi, S. D.; More, U. A.; Kulkarni, V. H.; Aminabhavi, T. M. Curr. Org. Chem. 2013, 17, 2279.

[2] Pinder, A. R. Nat. Prod. Rep. 1992, 9, 491.

[3] Tohme, R.; Darwiche, N.; Muhtasib, H. G. Molecules 2011, 16, 9665.

[4] Kumar, D.; Rawat, D. S. Oppor., Challenge Scope Nat. Prod. Med. Chem. 2011, 37, 213.

[5] Wiegard, A.; Hanekamp, W.; Griessbach, K.; Fabian, J.; Lehr, M. Eur. J. Med. Chem. 2012, 48, 153.

[6] Harrak, Y.; Rosell, G.; Daidone, G.; Plescia, S,; Schillaci, D.; Pujol, M. D. Bioorg. Med. Chem. 2007, 15, 4876.

[7] Gour, H. J.; Sanjay, J.; Sudershan, K. A.; Neelima, S. Bioorg. Med. Chem. Lett. 2005, 15, 3592.

[8] He, X. Y.; Zou, P.; Qiu, J. Y.; Hou, L.; Jiang, S. B.; Liu, S. W.; Xie, L. Bioorg. Med. Chem. 2011, 19, 6726.

[9] Montalbano, A.; Parrino, B.; Diana, P.; Barraja, P.; Carbone, A.; Spano, V.; Cirrincione, G. Tetrahedron 2013, 69, 2550.

[10] Padmaja, A.; Syamaiah, K.; Muralikrishna, A.; Padmavathi, V. Indian J. Chem. 2012, 51B, 285.

[11] Van Leusen, D.; Van Echten, E.; Van Leusen, A. M. J. Org. Chem. 1992, 57, 2245.

[12] Syamaiah, K.; Reddy, G. M.; Padmavathi, V.; Padmaja, A. Med. Chem. Res. 2014, 23, 3287

[13] Lan, L.; Zhan, X. P.; Qin, W. X.; Liu, Z. L.; Mao, Z. M. Heterocy- 
cles 2014, 89, 375.

[14] Lan, L.; Qin, W. X.; Zhan, X. P.; Liu, Z. L; Mao, Z. M. Anti-Cancer Agents Med. Chem. 2014, 14, 994.

[15] McCoy, T. A.; Maxwell, M.; Neuman, R. E. Cancer Res. 1956, 16, 979.

[16] Szkerke, M. Caner Treat Rep. 1976, 60, 347.

[17] Cox. P. J. Biochem. Pharmacol. 1979, 28, 2045.

[18] Zhao, R. L.; Fan, C. L.; Zhuo, R. X. Chem. J. Chin. Univ. 1989, 10, 605 (in Chinese).

(赵儒林, 范昌烈, 卓仁禧, 高等学校化学学报, 1989, 10, 605.)

[19] Wang, Q. D.; Xue, S. J.; Shen, J. F.; Cai, Z. J. Chin. J. Org. Chem. 2008, 28, 521 (in Chinese).

(王庆东，薛思佳，申杰峰，蔡志娟，有机化学, 2008, 28, 521.)

[20] Meng, G. R.; Li, J. J.; Wang, G. L.; Dong, M. J.; Zhang, Q. Chin. J. Org. Chem. 2013, 34, 155 (in Chinese).

(孟光荣, 李嘉俊, 王国林, 董孟杰, 张倩, 有机化学, 2013, 34, 155.)

[21] Othman, M. S.; Kawkab, Y. S.; Mowafaq, M. G. Int. J. Pharm.
Pharm. Sci. 2013, 5, 464.

[22] Wu, L.; Ye, H. Y.; Xiao, D. J. Chin. J. Pharm. 2004, 35, 67 (in Chinese).

(吴否, 叶和珏, 肖定军, 中国医药工业杂志, 2004, 35, 67.)

[23] Fukushima, M.; Nagao, Y.; Sano, S.; Yagi, T.; Yokoyama, K. Chem. Commun. 1997, 6, 559.

[24] Buck, I. M.; Black, J. W.; Cooke, T.; Dunstone, D. J.; Gaffen, J. D.; Griffin, E. P.; Harper, E. A.; Hull, R. A. D.; Kalindjian, S. B.; Lilley, E. J.; Linney, L. D.; Low, C. M. R.; McDonald, L. M.; Pether, M. J.; Robert, S. P.; Shankley, N. P.; Shaxted, M. E.; Steel, K. I. M.; Sykes, D. A.; Tozer, M. J.; Watt, G. F.; Walker, M. K.; Wright, L.; Wright, P. J. Med. Chem. 2005, 48, 6803.

[25] Gududuru, V.; Hurh, E.; Dalton, J. T.; Miller, D. D. J. Med. Chem. 2005, 48, 2584.

[26] Yang, M.; He, J. B.; Cheng, Y. X.; Jiang, S. Chin. J. Org. Chem. 2013, 33, 1319 (in Chinese).

(杨梅, 何江波, 程永现, 蒋晟, 有机化学, 2013, 33, 1319.)

(Li, L.; Lu, Z.) 